\title{
Acute kidney injury and 'nephrotoxins': mind your language
}

\author{
Authors: Mike Jones ${ }^{A}$ and Charles Tomson ${ }^{B}$
}

The introduction of the term 'acute kidney injury' (AKI) along with an international classification scheme, ${ }^{1}$ caused some initial confusion, but most clinicians and many patients now understand that the term 'injury' denotes damage to the internal workings of the kidney, rather than physical trauma. However, of greater concern is the use of the term 'nephrotoxic' to include drugs that are, in most settings, nephroprotective. We argue that this imprecise terminology, unfortunately adopted by the National Institute for Health and Care Excellence (NICE) among others, is potentially harmful, and that the terms 'nephrotoxin' and 'nephrotoxic' should not be used to describe haemodynamically mediated and fully reversible effects of some drugs on excretory function.

KEYWORDS: Acute kidney injury, nephrotoxins, ACEI, ARB

\section{Introduction}

In the last decade there has been increasing awareness that patients with 'acute kidney injury' (AKI) have a poorer prognosis than similar patients without AKI, whether the AKI develops in the community ${ }^{2}$ or in hospital ${ }^{3}$. This recognition has generated guidelines and quality improvement initiatives, including those from the National Institute for Health and Care Excellence $\left(\right.$ NICE $^{4}$ and from the Royal College of Physicians ${ }^{5}$. The NICE guideline includes angiotensin-converting enzyme inhibitors (ACEI) and angiotensin II receptor blockers (ARB) under the heading 'nephrotoxins', 4 and this terminology has been widely adopted and used in local care bundles. These drugs are, however, specifically indicated in heart failure, ${ }^{6}$ diabetic kidney disease, ${ }^{7}$ and proteinuric kidney disease ${ }^{8}$. These drugs are seldom truly nephrotoxic, although their effect on intrarenal haemodynamics can cause a reversible reduction in glomerular filtration rate (GFR), without structural damage. While this distinction may seem semantic, we argue that such lax terminology may have adverse effects on immediate and longer-term patient care.

Authors: ${ }^{A}$ consultant acute physician, University Hospital of North Durham, Durham, UK; ${ }^{\text {B }}$ consultant renal physician, Newcastle Upon Tyne Hospitals NHS Foundation Trust, Newcastle Upon Tyne, UK

\section{Nephrotoxicity}

There are, of course, many agents that are truly nephrotoxic. These should be used with caution in all patients and only when the riskbenefit ratio has been established and their use deemed necessary. This is especially true for patients who already have chronic kidney disease (CKD). Such nephrotoxic agents include aminoglycosides (eg gentamicin), iodinated radiocontrast agents, some antiretroviral drugs, and some chemotherapeutic agents (eg cisplatin). Beyond this group of agents there are those which, while generally well tolerated, may cause kidney damage due to unpredictable, idiosyncratic reactions. Interstitial nephritis can be caused by many antibiotics, by proton pump inhibitors, by aminosalicylates (eg mesalazine), and by non-steroidal anti-inflammatory drugs.

\section{Haemodynamically mediated changes in GFR}

The GFR of the normal kidney is usually maintained by autoregulation, even when the blood pressure changes. When renal perfusion pressure falls, glomerular filtration is regulated by prostaglandin-mediated vasodilation of the afferent arteriole and angiotensin-mediated vasoconstriction of the efferent arteriole. Autoregulation is impaired in CKD and in long-standing hypertension. In these conditions, a drop in systemic blood pressure can result in a fall in GFR, without any other marker of kidney damage (eg evidence of tubular injury). ${ }^{9,10}$ A profound and sustained decrease of blood pressure beyond the autoregulatory range will cause AKI. ${ }^{11}$ Preexisting low-flow states, including congestive cardiac failure (CHF), increase the risk of normotensive AKI. ${ }^{12}$

In the setting of reduced renal perfusion (eg bilateral renal artery stenosis: severe hypotension; severe intrarenal arteriosclerosis), non-steroidal anti-inflammatory drugs (NSAIDs) contribute to a fall in GFR by inhibiting vasodilatation of the afferent arteriole, and ACEIs and ARBs contribute to a fall in GFR by inhibiting vasoconstriction of the efferent arteriole. This is the reason for the common practice of suspending NSAIDs, ACEIs and ARBs in patients with, or at risk of, AKI. Whereas stopping NSAIDs is nearly certainly beneficial in this context, the evidence that stopping ACEIs or ARBs prevents AKI is very limited. Some go further and believe that their effects on efferent arteriolar tone may even improve tubular blood flow and thus limit tubular damage. ${ }^{13}$

\section{Management of patients on ACEI and ARB at risk of AKI}

The long-term benefits of ACEIs and ARBs have been proven in improving longevity in cardiac failure ${ }^{6}$ and preserving renal 
function in early diabetic kidney disease ${ }^{7}$ and in CKD with significant proteinuria. ${ }^{8}$ The effect of ACEI and ARB on glomerular perfusion (by reducing hyperfiltration) is the main mechanism by which ACEIs and ARBs slow progression of these types of kidney disease. The incidence of both cardiac failure and diabetes is increasing - so numerous patients are in receipt of these drugs.

\section{Sick day guidance and the use of ACEI/ARB}

Provision of 'sick day guidance', analogous to the 'sick day rules' provided to patients with diabetes on insulin treatment, has been widely adopted. Patients are often advised, either during a consultation or by provision of a leaflet or card, to stop taking ACEI and ARB (among other drugs) during 'acute illness', eg diarrhea, vomiting, or fever. While there may be benefits from this approach, telling patients that the medicine they are taking could actually be harmful may be associated with poorer therapeutic concordance with these medicines, and may even result in patients stopping drug treatment permanently. ${ }^{14}$ Like other complex interventions, provision of sick day guidance requires formal evaluation. In the interim, it is imperative that clear language should be used by those programmes that do suggest temporary cessation of therapy, and the terms 'nephrotoxic/nephrotoxin' should not be used in this setting.

\section{Temporary discontinuation of ACEI and ARB during} acute illness causing AKI

Management of patients with haemodynamic instability and AKI requires correction of both hypovolaemia and hypotension. Rational fluid replacement can only be achieved with repeated clinical assessment of fluid status. It is logical to suspend all antihypertensive drugs, including ACEI and ARB, during management of acute hypotension. Suspension of ACEI and ARB may cause a rise in GFR, as a result of altered glomerular haemodynamics; this can be of additional value if the GFR is already very low. ACEI, ARB and mineralocorticoid antagonists can also contribute to hyperkalaemia, which is an additional reason for temporary suspension. However, following restoration of adequate circulating volume, blood pressure, and recovery of GFR, the default should be that these drugs should be restarted, depending of course on the primary indication for their use but especially in patients with illness where ACEI or ARB therapy has been shown to be of particular benefit.

\section{Worsening renal function in the context of heart failure}

Acute decompensated heart failure is a common cause for hospital admission. In this context, worsening renal function is a poor prognostic marker. Management of these patients requires careful thought, and not reflex administration of intravenous fluids and cessation of ACEI or ARB. Administration of intravenous fluids to patients with heart failure, possibly prompted by deteriorating renal function, is associated with poorer outcomes. ${ }^{15}$ Increased systemic venous pressure as seen in cardiac failure can cause a reduction in GFR and, often to the surprise of trainee staff, decongestion by diuretics can result in an improvement in GFR. ${ }^{16}$ ACEI and ARB have been shown to improve long-term prognosis in heart failure with reduced ejection fraction (HFrEF). A metaanalysis of post hoc analyses from randomised controlled trials of ACEI or ARB in HFrEF demonstrated that the favourable impact on mortality conferred by ACEI or ARB was actually greater among those with worsening renal function than in those without, ${ }^{17}$ although no such benefit was seen in heart failure with preserved ejection fraction (HFpEF). It is acknowledged that these trials recruited patients with 'stable' heart failure and extrapolation to acute decompensated heart failure is not necessarily possible but the common practice of withdrawal of ACEI and ARB in response to a rise in serum creatinine concentration ${ }^{18}$ might well be harmful in this setting. In a large Scottish cohort study, acute pulmonary oedema was one of the commonest causes of early readmission after an episode of AKI; although no information on drug treatment was available in this cohort, it is possible that this association was caused by inappropriate withdrawal of ACEI, ARB, and diuretics. ${ }^{19}$

\section{Perioperative use of ACEI/ARB}

A meta-analysis of randomised and observational studies using propensity scoring demonstrated a marginally higher rate of AKI in patients whose ACEI or ARB were discontinued prior to surgery. ${ }^{20}$ A study of AKI after cardiac surgery showed that continuing ACEI/ ARB prior to cardiac surgery was associated with functional AKI (a rise in serum creatinine) but no change in markers of tubular injury. ${ }^{21}$ Therefore, routine perioperative cessation of ACE/ARB is unlikely to be beneficial.

\section{The 'Think Kidneys' programme}

The 'Think Kidneys' programme (www.thinkkidneys.nhs.uk) is a national quality improvement programme designed to reduce harm from AKI across England. It has provided guidance for healthcare practitioners to minimise the incidence of AKI and to provide guidance for optimal treatment of AKI, both in primary and secondary care. The programme does not endorse the use of the term 'nephrotoxic' for ACEIs or ARBs; it advises against the uncritical provision of 'sick day guidance'; and it has provided guidance to primary care on when to restart drugs stopped during hospital admissions with AKI. The decision to stop these drugs in patients at risk of, or with, AKI should be based on an individual assessment of risk and benefit. The decision should always be communicated in the summary of any in patient stay, enabling GPs to decide if or when to resume therapy after discharge. Patients should never be told that they have been receiving 'nephrotoxic' drugs unless this is actually the case. Further research on the optimal approach to drug treatment in patients with, or at risk of, AKI is urgently needed.

\section{Conflicts of interest}

Both authors are members of the 'Think Kidneys' steering group. There are no other conflicting interests.

\section{References}

1 Kidney Disease Improving Global Outcomes: KDIGO Clinical Practice Guideline for Acute Kidney Injury. Kidney International Supplements 2012;2:1-138.

2 Sawhney S, Fluck N, Fraser S et al. KDIGO-based acute kidney injury criteria operate differently in hospitals and in the community findings from a large population cohort. Nephrol Dial Transplant 2016;31:922-9. 
3 Chertow G, Burdick E, Honour M, Bonventre JV, Bates DW. Acute kidney injury, mortality, length of stay, and costs in hospitalized patients. J Am Soc Nephrol 2005;16:3365-70.

4 National Institute for Health and Care Excellence. Acute kidney injury: prevention, detection and management [CG169]. London: NICE, 2013.

5 Royal College of Physicians. Acute care toolkit 12: Acute kidney injury and intravenous fluid therapy. London: RCP, 2015.

6 National Institute for Health and Clinical Excellence. Chronic heart failure in adults: management [CG108]. London: NICE, 2010.

7 National Institute for Health and Care Excellence. Type 2 diabetes in adults: management [NG28]. London: NICE, 2015.

8 National Institute for Health and Care Excellence. Chronic kidney disease in adults: assessment and management [CG182]. London: NICE, 2014.

9 Ljungman S, Laragh JH, Cody RJ. Role of the kidney in congestive heart failure. Relationship of cardiac index to kidney function. Drugs 1990;39(Suppl 4):10-21; discussion 2-4.

10 Palmer BF. Renal dysfunction complicating the treatment of hypertension. N Engl J Med 2002;347:1256-61.

11 Liu YL, Prowle J, Licari E, Uchino S, Bellomo R. Changes in blood pressure before the development of nosocomial acute kidney injury. Nephrol Dial Transplant 2009;24:504-11.

12 Abuelo JG. Normotensive ischemic acute renal failure. N Engl J Med 2007:357:797-805.

13 Perazella MA, Coca SG. Three feasible strategies to minimize kidney injury in 'incipient AKI'. Nat Rev Nephrol 2013;9:484-90

14 Martindale AM, Elvey R, Howard S et al. Understanding the implementation of 'sick day guidance' to prevent acute kidney injury across a primary care setting in England: a qualitative evaluation. BMJ Open 2017;7:e017241.
15 Bikdeli B, Strait KM, Dharmarajan K et al. Intravenous fluids in acute decompensated heart failure. J Am Coll Cardiol Heart Fail 2015:3:127-33.

16 Afsar B, Ortiz A, Covic A et al. Focus on renal congestion in heart failure. Clin Kidney J 2016;9:39-47.

17 Beldhuis IE, Streng KW, Ter Maaten JM et al. Renin-angiotensin system inhibition, worsening renal function, and outcome in heart failure patients with reduced and preserved ejection fraction: a meta-analysis of published study data. Circ Heart Fail 2017;10:pii:e003588

18 Balasubramanian G, Al-Aly Z, Moiz A et al. Early nephrologist involvement in hospital-acquired acute kidney injury: a pilot study. Am J Kidney Dis 2011;57:228-34.

19 Sawhney S, Marks Fluck N et al. Acute kidney injury as a risk factor for unplanned 90-day hospital readmissions. BMC Nephrol 2017;18:9.

20 Cheungpasitporn W, Thongprayoon C, Srivali N et al. Preoperative renin-angiotensin system inhibitors linked to reduced acute kidney injury: a systematic review and meta-analysis. Nephrol Dial Transplant 2015;30:978-88.

21 Coca S, Garg A, Swaminathan M et al. Preoperative angiotensinconverting enzyme inhibitors and angiotensin receptor blocker use and acute kidney injury undergoing cardiac surgery. Nephrol Dial Transplant 2013;28:2787-99.

Address for correspondence: Dr Mike Jones, Acute Medical Unit, University Hospital of North Durham, Acute Medicine, North Road, Durham DH1 5TW, UK.

Email: mike.jones3@nhs.net 